\title{
The demonstration of two types of suppressor mechanism in leprosy patients and their contacts by quadruple skin-testing with mycobacterial reagent mixtures
}

\author{
PAMELA M NYE*, JANET E PRICE ${ }^{\dagger}$, \\ C R REVANKAR ${ }^{\ddagger}$, G A W ROOK* and J L STANFORD* \\ ${ }^{*}$ School of Pathology, Middlesex Hospital Medical School, \\ Riding House Street, London W1P 7LD; † Student at Sheffield \\ Medical School; ${ }^{\ddagger}$ Bombay Leprosy Project, 6/27 Amar Bhuvan, \\ Sion (East), Bombay 400 022, India
}

Received for publication 22 June 1982

\begin{abstract}
Summary A previous study in Nepal involving skin-testing simultaneously with four different mycobacterial reagents (three of them mixtures) revealed that the addition, in the same skin-test site, of pooled reagents from fast-growing mycobacteria locally suppressed the response to pooled slowgrowers. This finding has been confirmed in Bombay. It has also been shown that the addition of reagents prepared from certain fast-growing species to that prepared from slow-growers in one skin-test site will suppress the response to the same slow-grower reagent injected alone in the other arm. This type of suppression probably requires recognition of species-specific antigens of the fast-growing species concerned. The importance of the observations in relation to leprosy and possible mechanisms underlying them are discussed.
\end{abstract}

\section{Introduction}

In a preliminary investigation of responsiveness to shared and species-specific mycobacterial antigens carried out in a Nepalese leprosy hospital ${ }^{1}$ it was shown that patients had a markedly reduced response to both groups i and ii antigens as defined previously. ${ }^{2}$ It was also demonstrated that a proportion of people exhibited suppression of their responses to the group iv antigens of slowly growing species when injected as a mixture with the antigens of rapidly growing species. This suppression appeared to be specifically triggered by the group iv antigens of some of the rapidly growing species. 
The present study employs three of the skin-test reagents used in the preliminary work, together with a variable fourth reagent as described below. The purposes of this study were to determine whether the suppressor phenomenon found in Nepal was present in Bombay and to discover which of the rapidly growing species induced it.

\section{Materials and methods}

One hundred and ninety people were studied, including leprosy patients and healthy persons living in Bombay in the Kopri leprosy colony. Thirty-eight of the 190 had received BCG, although this made no appreciable difference to the results.

Each person received 4 simultaneous intradermal tests of $0.1 \mathrm{ml}$ volume containing $0.2 \mu \mathrm{g}$ protein, two on each forearm. Tests were read at $72 \mathrm{~h}$ and recorded as the mean of two diameters of the area of induration. Reactions of $2 \mathrm{~mm}$ or more were taken as positive. ${ }^{3}$

The following reagents were tested on every individual:

(1) A pool of equal volumes of sonicate preparations of 12 different slowly growing mycobacteria, each at a concentration of $1 \mathrm{mg}$ protein $/ \mathrm{ml}$ (reagent $\mathrm{SG})$.

(2) A similar pool of 12 preparations of rapidly growing mycobacteria (reagent FG).

(3) An equal mixture of the above two reagents (reagent $\mathrm{F} / \mathrm{S}$ ).

These three reagents, which were the same as those used in the preliminary study, ${ }^{1}$ were diluted with a tween containing borate buffer $(\mathrm{pH} 8.0)$ to $2 \mu \mathrm{g}$ protein $/ \mathrm{ml}$ for use.

At the fourth test site, 10 different reagents were tested, each on groups of approximately 20 people. These reagents consisted of equal volumes of reagent SG at $2 \mu \mathrm{g}$ protein $/ \mathrm{ml}$ and one of the following reagents also at $2 \mu \mathrm{g}$ protein $/ \mathrm{ml}$.

Chitin Neoaurumin

Diernhoferin Nonchromogenicin R507R

Duvalin Ranin

Flavescin Rhodesin

Gilvin Vaccin R887R

These are the constituents of FG, which contained in addition the duplicate preparations of Nonchromogenicin (R812R) and Vaccin (R859R). Reagents SG and FG were administered on the right forearm and F/S and the 4th reagents on the left forearm. 


\section{Results}

The overall percentage positivity and mean positive reaction size to each reagent are shown in Table 1 . They are arranged according to the contents of the 4th reagents. It can be seen from these results that both parameters of response to SG alone are dependent upon the content of the fourth reagent. When this contains Chitin, Diernhoferin, Rhodesin or Vaccin the mean percentage positivity to $\mathrm{SG}$ is $37 \%$ and the mean positive reaction size $8.1 \pm$ $5.4 \mathrm{~mm}$. When the fourth reagent includes Duvalin, Flavescin, Gilvin, Neoaurumin, Nonchromogenicin or Ranin, the mean percentage positivity to SG is $89 \%$ and the mean positive reaction size is $16.6 \pm 8.9 \mathrm{~mm}$. In the further analyses, reagents accompanying low responsiveness to SG are called group A and those with high responsiveness group B. The same effect is seen on the response to FG, but to a much lesser extent. Group A accompanies a $47 \%$

Table 1. Percentage positive responders and mean positive reaction sizes for each of the ten different reagents used at the fourth skin-test site

\begin{tabular}{|c|c|c|c|c|c|c|}
\hline & $\begin{array}{l}\text { Constituents of } \\
4 \text { th reagent }\end{array}$ & $\begin{array}{l}4 \text { th } \\
\text { reagent }\end{array}$ & SG & FG & $\mathrm{F} / \mathrm{S}$ & $\begin{array}{l}\text { No. in } \\
\text { group }\end{array}$ \\
\hline \multicolumn{7}{|c|}{ Group A } \\
\hline \multirow{2}{*}{\multicolumn{2}{|c|}{1 SG + Chitin }} & $85 \%$ & $30 \%$ & $30 \%$ & $60 \%$ & \\
\hline & & $10.4 \mathrm{~mm}$ & $7.6 \mathrm{~mm}$ & $4.4 \mathrm{~mm}$ & $11.5 \mathrm{~mm}$ & 20 \\
\hline \multirow[t]{2}{*}{2} & SG + Diernhoferin & $94 \%$ & $39 \%$ & $56 \%$ & $94 \%$ & \\
\hline & & $11.6 \mathrm{~mm}$ & $6.1 \mathrm{~mm}$ & $8.7 \mathrm{~mm}$ & $11.0 \mathrm{~mm}$ & 18 \\
\hline \multirow[t]{2}{*}{3} & $\mathrm{SG}+$ Rhodesin & $100 \%$ & $21 \%$ & $47 \%$ & $84 \%$ & \\
\hline & & $13.8 \mathrm{~mm}$ & $5.5 \mathrm{~mm}$ & $9.8 \mathrm{~mm}$ & $12.2 \mathrm{~mm}$ & 19 \\
\hline \multirow[t]{2}{*}{4} & $\mathrm{SG}+\mathrm{Vaccin}$ & $89 \%$ & $58 \%$ & $53 \%$ & $63 \%$ & \\
\hline & & $14.0 \mathrm{~mm}$ & $10.6 \mathrm{~mm}$ & $9.8 \mathrm{~mm}$ & $16.2 \mathrm{~mm}$ & 19 \\
\hline \multirow{2}{*}{\multicolumn{2}{|c|}{ Group A mean }} & $94 \%$ & $37 \%$ & $47 \%$ & $75 \%$ & \\
\hline & & $12.5 \mathrm{~mm}$ & $8.1 \mathrm{~mm}$ & $8.6 \mathrm{~mm}$ & $12.5 \mathrm{~mm}$ & \\
\hline \multicolumn{7}{|c|}{ Group B } \\
\hline \multirow[t]{2}{*}{5} & SG + Duvalin & $85 \%$ & $80 \%$ & $55 \%$ & $60 \%$ & \\
\hline & & $13.4 \mathrm{~mm}$ & $19.0 \mathrm{~mm}$ & $12.4 \mathrm{~mm}$ & $11.5 \mathrm{~mm}$ & 20 \\
\hline \multirow[t]{2}{*}{6} & $\mathrm{SG}+$ Flavescin & $89 \%$ & $84 \%$ & $58 \%$ & $79 \%$ & \\
\hline & & $13.7 \mathrm{~mm}$ & $19.8 \mathrm{~mm}$ & $11.1 \mathrm{~mm}$ & $12.2 \mathrm{~mm}$ & 19 \\
\hline \multirow[t]{2}{*}{7} & $\mathrm{SG}+$ Gilvin & $85 \%$ & $95 \%$ & $60 \%$ & $80 \%$ & \\
\hline & & $15.5 \mathrm{~mm}$ & $15.2 \mathrm{~mm}$ & $7.5 \mathrm{~mm}$ & $11.8 \mathrm{~mm}$ & 20 \\
\hline \multirow[t]{2}{*}{8} & $\mathrm{SG}+$ Neoaurumin & $82 \%$ & $88 \%$ & $47 \%$ & $71 \%$ & \\
\hline & & $10.9 \mathrm{~mm}$ & $15.0 \mathrm{~mm}$ & $7.3 \mathrm{~mm}$ & $15.2 \mathrm{~mm}$ & 17 \\
\hline \multirow[t]{2}{*}{9} & $\mathrm{SG}+$ Nonchromo- & $83 \%$ & $89 \%$ & $61 \%$ & $83 \%$ & \\
\hline & genicin & $12.3 \mathrm{~mm}$ & $15.1 \mathrm{~mm}$ & $11.5 \mathrm{~mm}$ & $12.7 \mathrm{~mm}$ & 18 \\
\hline & $\mathrm{SG}+$ Ranin & $95 \%$ & $95 \%$ & $74 \%$ & $58 \%$ & \\
\hline & & $14.4 \mathrm{~mm}$ & $16.2 \mathrm{~mm}$ & $10.5 \mathrm{~mm}$ & $14.3 \mathrm{~mm}$ & 19 \\
\hline \multirow{2}{*}{\multicolumn{2}{|c|}{ Group B mean }} & $87 \%$ & $89 \%$ & $60 \%$ & $72 \%$ & \\
\hline & & $13.5 \mathrm{~mm}$ & $16.6 \mathrm{~mm}$ & $10.0 \mathrm{~mm}$ & $12.8 \mathrm{~mm}$ & \\
\hline
\end{tabular}


Table 2. The effect of leprosy on the overall results

\begin{tabular}{llllll}
\hline Reagent & Contacts & TT & BT & BB/BL & LL \\
\hline Group A & & & & & \\
SG & $35 \%$ & $45 \%$ & $38 \%$ & $36 \%$ & $14 \%$ \\
& $6.9 \mathrm{~mm}$ & $8.4 \mathrm{~mm}$ & $8.8 \mathrm{~mm}$ & $7.0 \mathrm{~mm}$ & $14.5 \mathrm{~mm}$ \\
FG & $60 \%$ & $32 \%$ & $44 \%$ & $55 \%$ & $43 \%$ \\
& $8.7 \mathrm{~mm}$ & $8.7 \mathrm{~mm}$ & $5.0 \mathrm{~mm}$ & $12.0 \mathrm{~mm}$ & $9.0 \mathrm{~mm}$ \\
F/S & $70 \%$ & $59 \%$ & $88 \%$ & $91 \%$ & $86 \%$ \\
& $12.9 \mathrm{~mm}$ & $11.3 \mathrm{~mm}$ & $12.0 \mathrm{~mm}$ & $14.7 \mathrm{~mm}$ & $12.1 \mathrm{~mm}$ \\
4th & $100 \%$ & $86 \%$ & $88 \%$ & $91 \%$ & $100 \%$ \\
& $12.3 \mathrm{~mm}$ & $10.3 \mathrm{~mm}$ & $15.5 \mathrm{~mm}$ & $11.6 \mathrm{~mm}$ & $14.5 \mathrm{~mm}$ \\
Group B & & & & & \\
SG & $93 \%$ & $79 \%$ & $88 \%$ & $94 \%$ & $88 \%$ \\
& $16.3 \mathrm{~mm}$ & $13.3 \mathrm{~mm}$ & $21.2 \mathrm{~mm}$ & $17.1 \mathrm{~mm}$ & $16.1 \mathrm{~mm}$ \\
FG & $73 \%$ & $63 \%$ & $63 \%$ & $65 \%$ & $49 \%$ \\
& $8.1 \mathrm{~mm}$ & $8.1 \mathrm{~mm}$ & $9.6 \mathrm{~mm}$ & $11.4 \mathrm{~mm}$ & $11.0 \mathrm{~mm}$ \\
F/S & $73 \%$ & $74 \%$ & $75 \%$ & $81 \%$ & $63 \%$ \\
& $15.0 \mathrm{~mm}$ & $12.8 \mathrm{~mm}$ & $12.3 \mathrm{~mm}$ & $12.4 \mathrm{~mm}$ & $12.5 \mathrm{~mm}$ \\
4 th & $93 \%$ & $79 \%$ & $88 \%$ & $94 \%$ & $83 \%$ \\
& $14.0 \mathrm{~mm}$ & $11.6 \mathrm{~mm}$ & $14.4 \mathrm{~mm}$ & $14.0 \mathrm{~mm}$ & $13.1 \mathrm{~mm}$ \\
\hline
\end{tabular}

positivity to $\mathrm{FG}$ and a mean reaction size of $8.6 \pm 4.2 \mathrm{~mm}$, whereas group $\mathrm{B}$ accompanies a $60 \%$ positivity to $\mathrm{FG}$ and a mean reaction size of $10.0 \pm$ $5.1 \mathrm{~mm}$. The inclusion of groups A and B reagents do not seem to have any effect on responsiveness to $\mathrm{F} / \mathrm{S}$ and only marginally affect the mean positive reaction sizes to the 4 th reagents.

Table 2 shows the results obtained with groups A and B reagents according to whether those tested were healthy contacts or had leprosy. Reduced responsiveness to both SG and FG in the presence of group A, as compared with group B, is seen in all classes of persons tested. This is particularly the case amongst lepromatous patients of whom only 1 out of 7 (14\%) respond to SG in the presence of group A and 34 out of 41 (88\%) respond to SG in the presence of group B. Disease status has little effect in the presence of group $B$ reagents excepting small reductions of responsiveness to $F G$ and $F / S$ in lepromatous patients.

\section{Discussion}

At first sight the results from Bombay appear to bear little relationship to those from Nepal, ${ }^{1}$ doubtless due to the different environmental effects of the two places. The system of categorization used for the Nepal data is not readily applicable because the combination of reagents tested does not allow identification of individuals responding to the common antigens, and causes a false 
expansion of category 1 (positive to all four reagents). Nevertheless individuals failing to respond to one or more of the reagents can be taken as category 3 responders and for easier comparison with Nepal the individual results for these are shown in Table $3 a$ and $b$. Table $3 a$ is of persons tested with group A reagents and Table $3 \mathrm{~b}$ is of persons tested with group B reagents. There is a striking similarity between the results shown in Table $3 \mathrm{~b}$ and those in Table 3 of the Nepal study. Some of these points of similarity are shown in Table 4, as are points of difference with the results for recipients of group A reagents.

The kind of suppression discovered in Nepal in which the fast grower reagent $\mathrm{FG}$ is able to suppress locally the response to the slow grower reagent $\mathrm{SG}$ when mixed with it as reagent $\mathrm{F} / \mathrm{S}$ is equally present in Bombay amongst the recipients of group $\mathrm{B}$ reagents (halving the dose of a new tuberculin makes little difference to the response $\left.\operatorname{size}^{4}\right)$.

However, amongst the individuals showing suppression of F/S (Table $3 \mathrm{~b}$ ) only a minority show suppression of response to the 4th reagents. Thus it would not be correct to say that the species in the group B reagents, Mycobacterium duvalii, $M$. flavescens, $M$. fortuitum, M. gilvum, $M$. neoaurum and $M$. nonchromogenicum, are especially associated with the phenomenon. Amongst the results for the 55 persons receiving group B reagents and responding to all four tests 12 showed suppression of response to $\mathrm{F} / \mathrm{S}$ to half or less of the response to SG and thus also exhibit the phenomenon.

Included in Table 3 of the Nepal data were five individuals, indicated by an asterisk, for whose responses no explanation could be offered. One of these responded only to Burulin ${ }^{4}$ and is therefore irrelevant to the Bombay study. Two others were BL patients responding to FG and SG, but not $\mathrm{F} / \mathrm{S}$. These individuals and similar ones in the present study marked with asterisks in Table $3 \mathrm{~b}$ indicate that the addition of FG to SG can be suppressive whether or not there is a positive response to FG alone.

As in the Nepal study, this suppressory phenomenon is shown to a greater extent in leprosy patients than in their healthy contacts and therefore may be associated with disease. However, it must be borne in mind that in neither study was any attempt made to match by age, sex, race, etc., any of the groups studied, although this may become necessary in subsequent investigations.

Also marked with asterisks in Table 3 of the Nepal data were a lepromatous patient and a staff member responding to the $\mathrm{F} / \mathrm{S}$ mixture alone. Two individuals marked with double asterisks (one BT patient and one LL patient) in Table $3 \mathrm{~b}$ responded only to $\mathrm{F} / \mathrm{S}$ and the 4 th reagents. However, in Table $3 \mathrm{a}$ there are 18 such persons (4/17 contacts, 8/27 TT/BT patients, 3/7 BB/BL patients and $3 / 6$ LL patients). Nevertheless we still have no explanation of this effect.

The most striking feature of Table $3 \mathrm{a}$ and shown in Tables 1 and 4 is the dramatic reduction of responsiveness to $\mathrm{SG}$, when the 4th reagents included the group A species, $M$. chitae, $M$. diernhoferi, $M$. rhodesiae or $M$. vaccae. Not shown in Table $3 \mathrm{a}$ were the 15 persons receiving group A reagents and respon- 
Table 3(a) (and (b) opposite). Individual skin test responses expressed as mean diameters of induration $(\mathrm{mm})$ in persons of Category 3 receiving group A reagents (a) and group B reagents $(b)$

\begin{tabular}{|c|c|c|c|c|c|c|c|c|c|c|}
\hline & $\begin{array}{c}\text { Individual } \\
\text { No. }\end{array}$ & FG & SG & $\mathrm{F} / \mathrm{S}$ & 4 th & $\begin{array}{c}\text { Individual } \\
\text { No. }\end{array}$ & FG & SG & $\mathrm{F} / \mathrm{S}$ & 4 th \\
\hline \multirow[t]{21}{*}{ Contacts } & 17 & 0 & 0 & 0 & 8 & BT & 0 & 14 & 14 & 12 \\
\hline & 23 & 10 & 0 & 19 & 15 & 6 & 0 & 0 & 15 & $13 * *$ \\
\hline & 28 & 10 & $(2)$ & 14 & 18 & 16 & $(2)$ & 0 & 9 & 8 \\
\hline & 30 & 8 & 8 & 0 & $10 *$ & 22 & 5 & 0 & 27 & 17 \\
\hline & 33 & 0 & 0 & 0 & 9 & 26 & 0 & 3 & 12 & 15 \\
\hline & 37 & 0 & 10 & 0 & (2) & 166 & 0 & 0 & 18 & $33 * *$ \\
\hline & 38 & 9 & 19 & 0 & $11^{*}$ & 167 & 0 & 0 & 11 & $13 * *$ \\
\hline & 164 & 13 & 0 & 18 & 14 & 187 & 5 & 0 & 8 & 3 \\
\hline & 172 & 0 & 0 & 8 & $8 * *$ & 192 & 0 & 0 & 20 & $19 * *$ \\
\hline & 173 & 0 & 0 & 8 & $8^{* *}$ & 193 & 5 & 0 & 8 & 9 \\
\hline & 176 & 9 & 0 & 24 & 22 & 196 & 0 & 4 & 4 & 15 \\
\hline & 177 & 0 & 0 & 0 & 14 & & & & & \\
\hline & 179 & 4 & 0 & 13 & 18 & & & & & \\
\hline & 182 & 0 & 0 & 12 & $8 * *$ & & & & & \\
\hline & 189 & 11 & 0 & 14 & 15 & & & & & \\
\hline & 198 & 11 & 0 & 13 & 17 & & & & & \\
\hline & 200 & 0 & 0 & 12 & $10 * *$ & $\mathrm{BB} / \mathrm{BL} 5$ & 0 & 0 & 13 & $6 * *$ \\
\hline & & & & & & 21 & 20 & 0 & 26 & 12 \\
\hline & & & & & & 25 & 0 & 0 & 32 & $15^{* *}$ \\
\hline & & & & & & 178 & 6 & 0 & 12 & 15 \\
\hline & & & & & & 180 & 0 & 3 & 14 & 12 \\
\hline \multirow[t]{16}{*}{$\mathrm{TT}$} & 2 & 0 & 10 & 15 & 8 & 183 & 0 & 0 & 8 & $9 * *$ \\
\hline & 3 & 0 & 0 & 20 & $22 * *$ & 188 & 9 & 0 & 10 & 13 \\
\hline & 7 & 0 & 0 & 0 & 10 & & & & & \\
\hline & 8 & 3 & $(2)$ & 14 & 12 & & & & & \\
\hline & 10 & 3 & 0 & 0 & 11 & & & & & \\
\hline & 11 & 0 & 0 & 8 & $9 * *$ & & & & & \\
\hline & 13 & 0 & 0 & 0 & 7 & & & & & \\
\hline & 15 & 0 & 0 & 3 & $4 * *$ & LL 27 & 0 & 0 & 23 & $24 * *$ \\
\hline & 20 & 8 & 0 & 11 & 13 & 162 & 13 & 0 & 12 & 9 \\
\hline & 24 & 0 & 19 & 21 & 19 & 170 & 8 & 0 & 10 & 21 \\
\hline & 31 & 0 & 16 & 0 & 12 & 171 & 0 & 0 & 0 & 9 \\
\hline & 34 & 0 & 9 & 5 & 5 & 174 & 0 & 0 & 12 & $19 * *$ \\
\hline & 36 & 10 & 0 & 0 & 0 & 175 & 0 & 0 & 6 & $8 * *$ \\
\hline & 169 & 0 & 0 & 0 & 6 & & & & & \\
\hline & 194 & 0 & 8 & 10 & 8 & & & & & \\
\hline & 197 & 0 & 0 & 8 & $4 * *$ & & & & & \\
\hline
\end{tabular}

* and ${ }^{* *}$, see text for explanation. 
Table (b).

\begin{tabular}{|c|c|c|c|c|c|c|c|c|c|c|c|}
\hline & $\begin{array}{c}\text { Individual } \\
\text { No. }\end{array}$ & FG & SG & $\mathrm{F} / \mathrm{S}$ & 4 th & $\begin{array}{r}\text { Indiy } \\
1\end{array}$ & $\begin{array}{l}\text { idual } \\
\text { Jo. }\end{array}$ & $\mathrm{FG}$ & SG & $\mathrm{F} / \mathrm{S}$ & 4 th \\
\hline \multirow[t]{9}{*}{ Contacts } & 41 & 7 & 12 & 0 & $11 *$ & $\mathrm{BB} / \mathrm{BL}$ & 47 & 24 & 0 & 0 & 10 \\
\hline & 105 & 8 & 11 & 0 & $17^{*}$ & & 49 & 14 & 17 & 0 & 12 \\
\hline & 115 & 0 & 20 & 0 & 10 & & 54 & 0 & 27 & 12 & 14 \\
\hline & 138 & 0 & 21 & 13 & 18 & & 58 & 0 & 12 & 11 & 8 \\
\hline & 152 & 0 & 15 & 14 & 14 & & 60 & 0 & 30 & 20 & 0 \\
\hline & & & & & & & 76 & 0 & 13 & 10 & 10 \\
\hline & & & & & & & 77 & 0 & 13 & 11 & 10 \\
\hline & & & & & & & 110 & 10 & 15 & 0 & $14^{*}$ \\
\hline & & & & & & & 113 & 0 & 14 & 8 & 12 \\
\hline \multirow[t]{10}{*}{ TT } & 42 & 13 & 13 & 0 & $14^{*}$ & & 133 & 0 & 34 & 12 & 33 \\
\hline & 44 & 7 & 0 & 10 & 14 & & 146 & 0 & 17 & 0 & 10 \\
\hline & 94 & 0 & 9 & 3 & 7 & & 148 & 0 & 18 & 0 & 3 \\
\hline & 137 & 0 & 9 & 8 & 13 & & 153 & 0 & 12 & 13 & 11 \\
\hline & 140 & 0 & 15 & 8 & 9 & & & & & & \\
\hline & 150 & 0 & 10 & 13 & 0 & & & & & & \\
\hline & 160 & 3 & 12 & 0 & $15^{*}$ & & & & & & \\
\hline & & & & & & LL & 45 & 0 & 27 & 0 & 16 \\
\hline & & & & & & & 46 & 0 & 0 & 3 & $8 * *$ \\
\hline & & & & & & & 51 & 0 & 23 & 0 & 14 \\
\hline \multirow[t]{21}{*}{ BT } & 108 & 11 & 11 & 0 & $18^{*}$ & & 52 & 14 & 12 & 0 & $11 *$ \\
\hline & 130 & 0 & 14 & 11 & 15 & & 57 & 0 & 10 & 6 & 20 \\
\hline & 135 & 0 & 10 & 0 & 0 & & 59 & 0 & 30 & 20 & 0 \\
\hline & 157 & 0 & 0 & 8 & $6 * *$ & & 63 & 0 & 18 & 14 & 14 \\
\hline & & & & & & & 66 & 0 & 20 & 0 & 12 \\
\hline & & & & & & & 71 & 0 & 0 & 0 & 9 \\
\hline & & & & & & & 78 & 0 & 39 & 32 & 27 \\
\hline & & & & & & & 80 & 0 & 12 & 10 & 11 \\
\hline & & & & & & & 96 & 0 & 17 & 12 & 11 \\
\hline & & & & & & & 100 & 0 & 12 & 7 & 16 \\
\hline & & & & & & & 101 & 0 & 8 & 0 & 17 \\
\hline & & & & & & & 103 & 0 & 8 & 0 & 8 \\
\hline & & & & & & & 114 & 0 & 0 & 0 & 6 \\
\hline & & & & & & & 118 & 5 & 14 & 0 & $0 *$ \\
\hline & & & & & & & 121 & 0 & 14 & 10 & 14 \\
\hline & & & & & & & 122 & 18 & 2 & 0 & $0 *$ \\
\hline & & & & & & & 129 & 5 & 0 & 0 & 0 \\
\hline & & & & & & & 139 & 0 & 11 & 0 & 10 \\
\hline & & & & & & & 143 & 0 & 10 & 0 & 0 \\
\hline & & & & & & & 154 & 0 & 14 & 13 & 6 \\
\hline & & & & & & & 84 & 0 & 6 & 0 & 0 \\
\hline
\end{tabular}

* and ${ }^{* *}$, see text for explanation. 
Table 4. Similarities and differences between results for Nepal and Bombay according to the 4 th reagent groups.

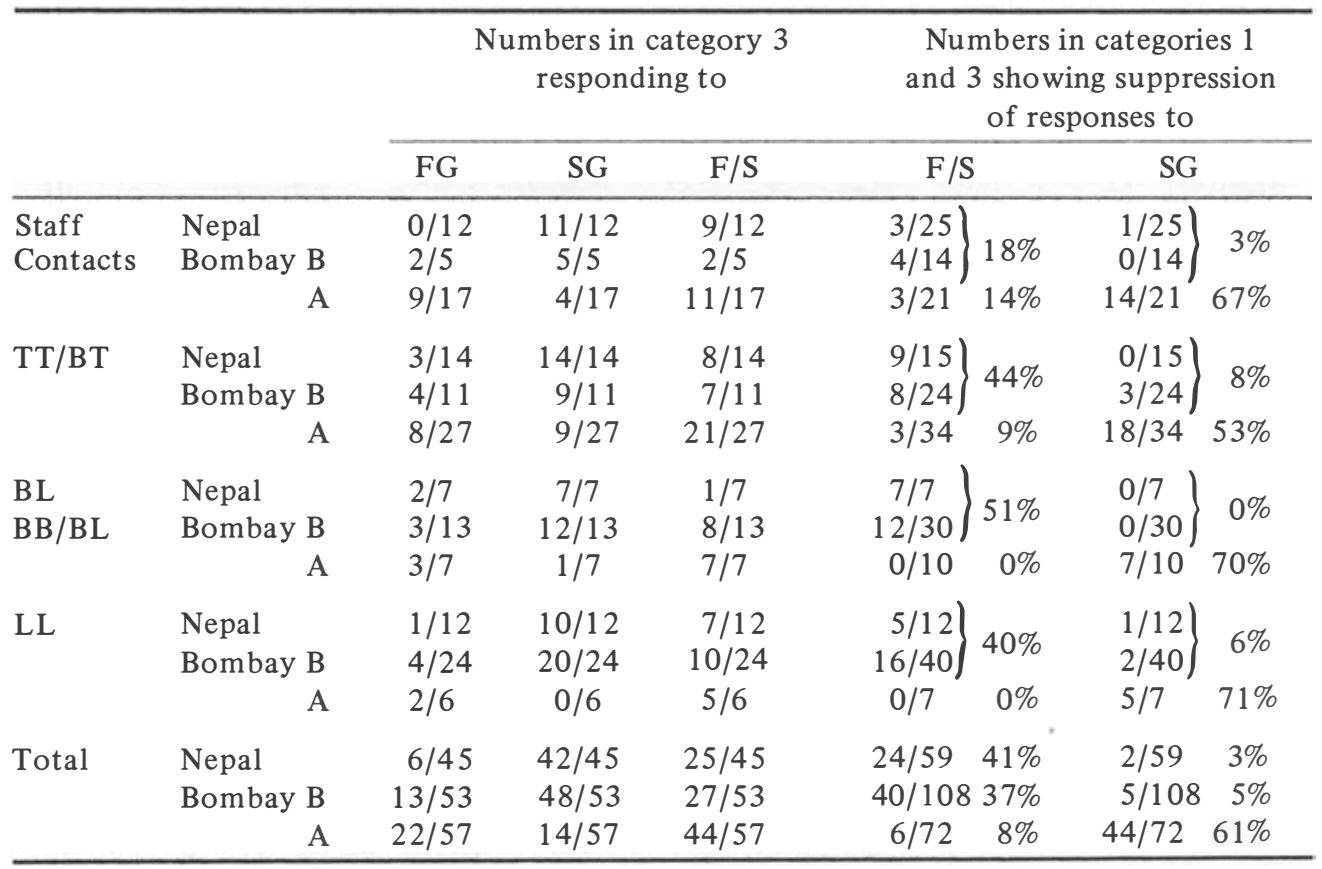

ding to all four tests. Six of these had SG responses which were half or less of those to $\mathrm{F} / \mathrm{S}$. These individuals can be taken as showing the same phenomenon. It is striking, however, that as with group B reagents responses to the 4th reagents themselves are only suppressed in a minority of cases.

This use of reagent mixtures has demonstrated two distinct types of suppressor mechanism. The first acts locally, and does not affect other skin-test sites. The second mechanism is associated with group A, but not group B reagents. Thus it seems that when the antigens of slow growers are presented together with antigens of the group A species in one arm, a suppressor mechanism results in failure to respond to the same slow-grower antigens given alone in the other arm. That this mechanism should be operative within $72 \mathrm{~h}$ makes it much more probable that an existing system has been triggered than that a new system has been induced. The most likely mechanism would seem to be triggering by the fast grower of some form of suppressor cell ${ }^{5}$ perhaps resulting in release of soluble suppressor factors. ${ }^{6}$ These could either recognize the antigens of slow growers, or specifically block a particular kind of response. Since the response blocked is exclusively that to SG and slightly predominates in BB to LL rather than TT/BT patients, it seems likely that the effect is against Kochtype responses. ${ }^{7-9}$

On the other hand, the suppression of response to $\mathrm{F} / \mathrm{S}$ seen in Nepal and in the presence of group B reagents in Bombay, appears to be antigen directed 
rather than response type directed and is greatest in the patient groups (57/ $128,45 \%)$ rather than staff/contact individuals $(7 / 39,18 \%)$.

The question arises whether these phenomena are consequences of particular types of leprosy or prerequisites for them.

Many other important questions about these suppressor phenomena remain unanswered. Amongst the most pressing is the question of sensitization by the fast growers. One interpretation of the group B results would be that the species concerned do not exert any effect because their species-specific (group iv) antigens are not recognized and therefore the responses to the 4th reagents approximate to those to SG alone. On this basis the response to group A 4th reagents might be to the species-specific antigens of the fast growers themselves and therefore of Listeria type. ${ }^{7,8}$

If this is true then the effect of each species will depend upon the pattern of sensitization of the persons tested and this would explain why not everyone shows the suppressor phenomena. Similarly, different species will behave as groups A and B in different parts of the world. One species we can be sure of in the presence of active leprosy patients the world over is M.leprae and its effects will be investigated in future studies.

Work in progress in our laboratory and elsewhere (C Brown, I. Brown and M J Shield, unpublished observations) suggests that such suppressor phenomena may be primed by oral intake of mycobacteria. Large numbers of mycobacteria are known to be present in water supplies in Bombay. (J. Kazda, personal communication) just as they were in nineteenth century Norway. ${ }^{10}$ The implied importance of these phenomena in relation to the development of leprosy requires further investigation.

In conclusion, two types of suppression have been demonstrated in our quadruple skin-testing system in Bombay. One of these is similar to that already demonstrated in Nepal. The second type, which suppresses skin-test responses at distant sites, appears to be associated with recognition of speciesspecific antigens of 4 of the 10 fast-growing species investigated.

\section{Acknowledgements}

We would like to thank Dr R Ganapati, Director of the Bombay Leprosy Project and Dr J K Maniar of the Grant Medical College, Bombay for their support and encouragement of this work. Our thanks are also given to Lepra for the financial support that made this study possible.

\section{References}

1 Stanford JL, Nye PM, Rook GAW, Samuel N, Fairbank A. A preliminary investigation of 
the responsiveness or otherwise of patients and staff of a leprosy hospital to groups of shared or species specific antigens of mycobacteria. Lepr Rev 1981; 52: 321-7.

Stanford JL, Grange JM. The meaning and structure of species a s applied to mycobacteria. Tubercle 1974; 55: 143-52.

${ }^{3}$ Shield MJ, Stanford JL, Paul RC, Carswell JW. Multiple skin-testing of tuberculosis patients with a range of new tuberculins and a comparison with leprosy and Mycobacterium ulcerans infection. J Hyg 1977; 78: 331-48.

${ }^{4}$ Stanford JL, Revill WDL, Gunthorpe WJ, Grange JM. The production and preliminary investigation of Burulin, a new skin-test reagent for Mycobacterium ulcerans infection. J Hyg 1975; 74: 7-16.

5 Rook GAW. Suppressor cells of mouse and man. What is the evidence that they contribute to the aetiology of the mycobacterioses? Lepr Rev 1982; 53: 306-312.

6 Asherson GL, Zembala M. The role of the T-acceptor cell in suppressor systems: antigen specific T-suppressor factor acts via a $\mathrm{T}$-acceptor cell; this releases a non-specific inhibitor of the transfer of contact sensitivity when exposed to antigen in the context of I-J. Ann NY Acad Sci (in press).

7 Stanford JL, Shield MJ, Rook GAW. How environmental mycobacteria may predetermine the protective efficacy of BCG. Tubercle $1981 ; 62$ : 55-62.

8 Rook GAW, Bahr GM Stanford JL. The effect of two distinct forms of cell-mediated response to mycobacteria on the protective efficacy of BCG. Tubercle 1981; 62: $63-8$.

9 Stanford JL. A mycobacteriologist's view of the immunology of leprosy. Bull Inst Pasteur $1981 ; 79: 261-73$.

10 Irgens LM, Kazda J, Muller K, Eide GE. Conditions relevant to the occurrence of acid fast bacilli in Sphagnum vegetation. Acta Path Micr Scand, Sect B 1981; 89: 41-7. 